\title{
Distributed Dynamic Spectrum Access in Multichannel Random Access Networks with Selfish Users
}

\author{
Ali Ozyagci, Jens Zander \\ Royal Institute of Technology \\ Wireless@KTH \\ Stockholm, Sweden \\ Email: \{ozyagci, jenz\}@kth.se
}

\begin{abstract}
Dynamic spectrum allocation schemes enable users to share spectrum resources by exploiting the variations in spectrum demand over time and space. Performing dynamic spectrum allocation centrally can be prohibitively complex. Therefore distributed schemes in which users can access the available channels independently may be preferable to centralized allocation. However, in distributed dynamic spectrum access, the lack of central coordination makes it difficult to utilize the system resources efficiently. Furthermore, if some or all of the users decide to deviate selfishly from the commonly agreed access procedure, this may have a decisive effect on system performance. In this paper we investigate the effect of incomplete information and selfish behavior on system performance in wireless access systems. We extend previous work by studying a distributed multichannel wireless random access system. Using a gametheoretic approach, we analyze the behavior of users in the selfish system and derive the transmission strategies at the Nash equilibrium. Our results show that lack of information leads to substantial degredation in performance of cooperative systems. We also show that there is a large incentive for selfish behavior in such cooperative systems. Selfish behavior of all users, however, causes further performance degradation, particularly in high load settings.
\end{abstract}

\section{INTRODUCTION}

Dynamic spectrum access (DSA) schemes are gaining importance with the emergence of cognitive radio and "smart" radio concepts. Such sophisticated radio equipment capable of accessing different segments of spectrum along with narrowband modulation capabilities make various forms of spectrum sharing feasible [1]. For instance, reusing temporarily unused spectrum, so called whitespace, in the form of an overlay system has been proposed in literature.

Performing dynamic spectrum allocation centrally in such systems would be overwhelmingly complex and possibly NPhard as mentioned in [2]. Problems with system complexity can be avoided through distributed spectrum sharing mechanisms in which users access the medium either by following a set of rules or at their own discretion. These distributed mechanisms can be cooperative, where nodes try to collectively maximize the system performance, or they can be noncooperative, where the nodes selfishly try to maximize their own benefits only. The study of cooperative random access systems dates back to the 70's with the ALOHA systems as most popular representatives. These schemes are analytically tractable and despite their simplicity, they capture many of the characteristics of wireless random access systems.

Studies regarding random access systems where the users are selfish have so far been confined to single-channel settings [3]-[6]. In [3], [4] the authors analyze a single channel ALOHA system using game theory and derive the Nash equilibrium strategies. In [5], [6] the authors consider a single channel ALOHA system with packet capture under incomplete information assumption, that is, a user has exact knowledge of only its own channel conditions and it has solely statistical knowledge about the channel conditions of the other users. They show that users' best response is a threshold strategy where users transmit on the channel if their pathgain is above a certain level.

One extension of this concept are ALOHA schemes with multiple channels which better captures the fundamental properties of DSA. In [7] a Markovian analysis of such a multichannel ALOHA system was given where throughput and stability of the system is considered. In this paper we extend the work in [5], [6] to a multi-channel random access (MRA) system in which users have incomplete information. We first analytically calculate the optimal transmission strategy for a selfish MRA system in which each user tries to maximize its own utility. Then we compare the performance of three systems in terms of utilities: a selfish MRA system, a cooperative MRA system where users try to maximize the sum of their utilities using incomplete information, and a scheduling system where users have complete information of system conditions and act cooperatively to attain maximum possible sum utility in the system. By comparing the cooperative and scheduling systems we observe the degradation in system performance due to lack of complete information. We also determine the "the price of anarchy", i.e. the performance loss in a selfish system compared to a cooperative system.

Comparison between selfish and cooperative systems shows the price of anarchy in the system, which is defined as the ratio of maximum sum utility achievable in a system to the sum utility achieved by selfish users. We also compare the performance of a cooperative system to the same system where 
we assume there is only one selfish user in order to observe the incentives for a user to act selfishly in a cooperative system.

\section{System Model AND Assumptions}

In what follows, we compare four variations of slotted distributed MRA systems. In neither of these systems is there a central entity to dictate users what action they should take. In every time slot, the users decide by themselves whether to transmit or to wait. In the selfish system that we consider, users have incomplete information about the channel conditions, which means that a user knows its own exact channel conditions (e.g. pathloss), but it has only statistical knowledge (e.g. distribution) of the channel conditions of other users in the system. Based on this information, in each time slot a selfish user decides on its action in order to maximize its own benefits, by taking into account the actions it expects the other users to take in that time slot. In the cooperative system, the users also have incomplete information about channel conditions, similar to the selfish case. But unlike the selfish case, the cooperative users choose their actions to maximize the sum utility in the system. The users in the scheduling system are also cooperative users but in contrast to the cooperative system they have complete knowledge of each user's channel conditions. Thus, every user is able to calculate by itself which group of users should transmit in a time slot in order to maximize sum utility, and every user transmits or waits according to this calculation. In the cooperative system with one selfish user we assume all users except for one behave cooperatively, and only one user behaves selfishly. We will refer to the selfish user in this system as "the cheater".

In order to compare the performance of the cooperative and selfish systems we use the user utility [5], [6]. The utility, or the user-perceived benefit, is a function of the throughput achieved and the energy spent by the user. The general definition of utility we use in this work is the following: When a user transmits a packet, it gains a benefit if this packet is successfully received. In case of a failed transmission there is no benefit for the user, obviously. For each transmission, regardless of success, the user spends some energy to send the packet, therefore an energy cost is associated with each transmission. Utility can be defined generally as the difference between the expected benefit from a transmission and the cost of this transmission. In this work we focus on the average sum utility per channel, which would reflect the efficiency of channel utilization in the system, thus indicate the performance of the system as a whole. A second metric that will be used is average user utility because this metric indicates the utility that a user gets from the system, so it reflects the system's performance from the user's point of view.

To simplify our analysis, but without loss of generality, we make the following assumptions. Time is divided into slots and users transmit their packets on the channels within these slots. The channels we consider in MRA system are collision channels, that is, when more than one user transmits on the same channel in the same time slot, the packets of all the users who transmit on that channel will be lost. For simplicity we do not consider multipacket transmission or power capture. Furthermore, users are assumed to have full buffers so they always have a packet to transmit.

In the MRA system we consider there are $\mathrm{N}$ users and $\mathrm{K}$ channels. We assume that users, whether selfish or cooperative, are rational; they try to maximize their own utilities or the sum of utilities in the system respectively. The users know the total number of users $(N)$ in the system; they may be provided with this information by means of a common control channel or they can estimate this using the pseudo-Bayesian algorithm explained in [8].

When a particular user $n$ transmits a packet on channel $k$, it incurs a normalized transmision cost of $e_{n k}$. If the transmission is successful, it also gains a normalized utility of 1 . Let $T_{n k}(\mathbf{S})$ denote the probability that user $n$ successfully transmits a packet on channel $k$, where $\mathbf{S}$ is a vector that denotes the actions of all the users in the system. Then we can express the expected utility that user $n$ obtains from transmitting on channel $k$ as the following:

$$
\begin{aligned}
U_{n k}(\mathbf{S}) & =T_{n k}(\mathbf{S})\left(1-e_{n k}\right)+\left(1-T_{n k}(\mathbf{S})\right)\left(-e_{n k}\right) \\
& =T_{n k}(\mathbf{S})-e_{n k}
\end{aligned}
$$

Note that $e_{n k}$ associated with the transmission of a packet represents the perceived expense of this transmission by the user. This cost can be a function of the propagation loss associated with the channel conditions, remaining battery capacity of the terminal, or a similar measurement (delay, noise, etc). We assume that this transmission cost is normalized by the satisfaction that the user receives from successful transmission. Therefore, this quantity depends on the user preferences and there may be different ways to model this cost. In this work, we define the normalized transmission cost $e_{n k}$ in a given slot to be the ratio of the pathloss that user $n$ experiences on channel $k$ in that slot to the expected pathloss at the nominal communication range $r_{0}$ of the system, for instance the cell border in a cellular system. For a user who is at a distance $r$ to its receiver, the normalized transmission cost is

$$
e_{n k}=c \cdot\left(\frac{r}{r_{0}}\right)^{\alpha} \frac{1}{S \cdot R}
$$

where $\alpha$ denotes the pathloss exponent and $D \triangleq\left(r / r_{0}\right)^{\alpha}$ is the distance dependent component of the transmission cost. The shadow fading component $S$ is a lognormally distributed random variable with unit mean and standard deviation $\sigma$, and the fast-fading component $R$ is an exponentially distributed random variable with unit mean. To approximate an urban environment we assumed $\alpha=3$ and $\sigma=4$. The constant $c$ is a scaling factor. We assume the normalized utility at nominal communication range to be equal to the normalized transmission cost, therefore it is reasonable to write $c=1$.

We further assume that in the selfish and cooperative systems each user has complete information about their own channel conditions but only has statistical knowledge about the channel conditions of other users. So, a user would exactly know its own transmission costs on different channels in 
a given slot $\left(e_{n k}\right)$; the user could obtain these costs from relevant channel measurements. Furthermore, when the users are mobile, it is reasonable to assume that the distribution of the transmission costs are the same for every user-channel pair in the system, that is, $F_{n k}(x)=F(x), \forall n, k$. Thus, the user would be able to obtain the statistical distribution of the transmission costs $(F(x))$ by observing its own channel conditions over a number of slots. So, it would be possible for a user to obtain all this information locally, eliminating the need for receiving this information from a central entity. It is also important to note that, although the distribution of the transmission costs are the same for all users and channels, per-slot realizations of these costs will be different. In some realizations the transmission costs of some channels could be greater than the utility of successful transmission, thus rendering these channels unavailable to some users. In this way, the model we use captures the dynamic channel availability nature of DSA systems.

We assume that the distance dependent component of pathloss $(D)$ and shadow fading $(S)$ are independent of the carrier frequency, so in a given realization a user experiences the same $D$ and $S$ across the $K$ channels in the system. Nevertheless $R$ will be different because we assume that the $K$ channels in the system, whether they come from adjacent bands or separate bands, will display different propagation characteristics due to fast-fading. We also assume that channel coherence time is longer than the slot duration so that fast-fading component is essentially constant within a slot. Shadow fading components of each user is also uncorrelated in our assumptions and likewise fast fading components are uncorrelated for each user and channel.

\section{Game Theoretic Analysis}

In order to analyze the user behavior and performance of the systems we formulate the MRA system with selfish users as a non-cooperative game and we subsequently obtain the expressions for the transmission probability and the transmission cost threshold at the Nash equilibrium.

\section{Game Theoretic Solution to the Selfish System}

As laid out previously, each user knows the following: 1.Its own transmission costs $\left(e_{n k}\right)$ on each of the $K$ channels. 2.Statistical distribution of transmission costs, i.e. the CDF $F(x)$, which is the same for all of the $N$ users on all $K$ channels. 3.The number of users in the system, $N$. Then, multichannel random access game with incomplete information (MRAG-I) is defined in the following way:

- Players: The players are the $\mathrm{N}$ users $(n \in \mathcal{N}, \mathcal{N}=$ $\{1, \ldots, N\})$ who randomly access the $K$ channels in the system $(k \in \mathcal{K}, \mathcal{K}=\{1, \ldots, K\})$.

- Strategies: The strategy of a user $n$ is its probabilities of transmission on the $\mathrm{K}$ channels: $\mathbf{S}_{n}=$ $\left(p_{n 0}, p_{n 1}, p_{n 2}, \ldots, p_{n k}, \ldots, p_{n K}\right)$. The probability that user $\mathrm{n}$ chooses to wait in a slot is denoted by $p_{n 0}$. We can represent the set of strategies that all the users employ as $\mathbf{S}=\left(\mathbf{S}_{1}, \mathbf{S}_{2}, \ldots, \mathbf{S}_{n}, \ldots, \mathbf{S}_{N}\right)$.
- Utilities: As given in (1) the expected utility of user $n$ from transmitting on channel $k$ is its success probability minus its transmission cost on that channel. The probability that user $n$ successfully transmits a packet on channel $k$ is the the probability that no other user transmits on channel $k$ in the same slot, which is given by

$$
T_{n k}(\mathbf{S}) \triangleq \prod_{\substack{i=1 \\ i \neq j}}^{N}\left(1-p_{i k}\right)
$$

The overall utility that user $n$ obtains from its strategy $\mathbf{S}_{n}$ is then $U_{n}(\mathbf{S})=p_{n 0} \cdot 0+\sum_{k=1}^{K} p_{n k}\left(T_{n k}(\mathbf{S})-e_{n k}\right)$. Here the weight of $p_{n} 0$ is zero because the utility associated with waiting is zero.

In order to determine the behavior of these selfish and cooperative users, we make the following two observations:

1. Identical statistical behavior: Under the assumption that the distribution of transmission costs are the same for every user on every channel, long term behavior of all the users at the Nash equilibrium will be the same. In other words, at Nash equilibrium of MRAG-I the transmisson probabilities of each user on each channel will be the same: $p_{n k}^{*}=p_{m l}^{*}=$ $p^{*}, \forall n, m \in \mathcal{N}, \forall k, l \in \mathcal{K}$. Note that in general $p_{n 0} \neq p^{*}$. In view of this observation, using (3) we can write $U_{n k}(\mathbf{S})=$ $\left(1-p^{*}\right)^{N-1}-e_{n k}$. The utility of waiting is $U_{n 0}=0$.

2. Threshold strategy: Note that, at the beginning of a time slot each user decides if and on exactly which channel it will transmit. However, due to incomplete information constraint, user $n$ does not know on which channel other users will transmit, so other users' actions are probabilistic from the point of user $n$. Now, by rewriting $U_{n}(\mathbf{S})$ in open form

$$
\begin{aligned}
U_{n}(\mathbf{S})= & p_{n 0} U_{n 0}+p_{n 1} U_{n 1}+\cdots+p_{n k} U_{n k}+ \\
& +\cdots+p_{n K} U_{n K} \\
= & p_{n 0} \cdot 0+p_{n 1}\left(\left(1-p^{*}\right)^{N-1}-e_{n k}\right)+ \\
& +\cdots+p_{n K}\left(\left(1-p^{*}\right)^{N-1}-e_{n K}\right)
\end{aligned}
$$

we can observe that in order to maximize $U_{n}(\mathbf{S})$ user $n$ should transmit with probability 1 on the channel that gives the highest utility, or wait if all $U_{n k}, k \in\{1, \cdots, K\}$ are negative. In other words, user $n$ should transmit with probability 1 on the channel with the smallest transmission cost which is less than the success probability of user $n$, and if there is no such channel user $n$ should wait. The transmission cost threshold is thus the success probability: $e^{*}=\left(1-p^{*}\right)^{N-1}$.

Considering these two observations, we can deduce that user $n$ transmits on channel $k$ if and only if $e_{n k}=\min \left(e_{n j}\right), j \in$ $\{1, \cdots, K\}$ and $\left(1-p^{*}\right)^{N-1}>e_{n k}$. So we can calculate the probability that user $n$ transmits on channel $k$ as:

$$
\begin{aligned}
p_{n k}^{*}= & p^{*}=P[\underbrace{e_{n k}=\min \left(e_{n j}\right), j \in\{1, \cdots, K\}}_{\text {event A }} \\
& \wedge \underbrace{\left(1-p^{*}\right)^{N-1}>e_{n k}}_{\text {event } \mathrm{B}}] \\
= & P[A \cap B]=P[B \mid A] \cdot P[A]
\end{aligned}
$$


According to the pathloss model we use, for user $n$ the transmission costs $e_{n k}$ have the same distance dependent $(D)$ and shadow fading $(S)$ components but the fast-fading components $(R)$ are independent and identically distributed, therefore $P[A]$ is the event that $e_{n k}$ is the minimum of $K$ i.i.d. random variables, which is $1 / K$. The second part of the expression, $P[B \mid A]=P\left[\left(1-p^{*}\right)^{N-1}>e_{n k} \mid A\right]=P\left[e_{n k}<\right.$ $\left.e^{*} \mid A\right]$ is the probability that $e_{n k}$ is less than the transmission cost threshold, given that it is the smallest of $K$ identically distributed transmission costs. We can express $P[B \mid A]$ as $F_{K}\left(e^{*}\right)$ where $F_{K}(x)$ is the $\mathrm{CDF}$ of the minimum of $K$ identically distributed random variables, all drawn from the distribution $F(x)$. It is important to note that in our model $e_{n k}$ and $e_{m j}$ are identically distributed but they are not independent although $e_{n k}$ and $e_{n j}$ are i.i.d. Therefore we cannot express $F_{K}(x)$ as $1-(1-F(x))^{K}$. Nevertheless, $F_{K}(x)$ can be obtained empirically by observing the minimum transmission costs of $\mathrm{K}$ channels, or it can be calculated numerically.

Having obtained the expressions for $P[B \mid A]$ and $P[A]$ we can calculate $p^{*}$ as:

$$
\begin{aligned}
p^{*} & =P[B \mid A] \cdot P[A]=F_{K}\left(e^{*}\right) \cdot \frac{1}{K} \\
& =F_{K}\left(\left(1-p^{*}\right)^{N-1}\right) \frac{1}{K}
\end{aligned}
$$

From (8) we can calculate the transmission probability at the Nash equilibrium, $p^{*}$, and in turn the transmission cost threshold at Nash equilibrium $e^{*}$ which is equal to $\left(1-p^{*}\right)^{N-1}$.

\section{Simulation Results}

In this section we present numerical results in order to compare the performance of the four systems in question; namely the selfish, cooperative, cooperative with one cheater, and scheduling systems.

\section{Methodology}

We simulated the performance of these four systems by generating numerous realizations for various numbers of channel and user combinations. In each realization (snapshot) we simulated one time slot of a given system type and $(N, K)$ combination. We randomly placed the users across the system area and calculated their normalized transmission costs $\left(e_{n k}\right)$, which we obtained from the random channel conditions of the user, as explained in section 2 . Then we observed the number of successful transmissions in this snapshot and calculated the utilities according to the user utility definition given in (1) in order to obtain statistics of the performance of the system in question.

\section{Transmission strategies}

In each of the systems, the users employ different transmission strategies to reach their objectives. In the selfish system the users try to maximize their individual utilities and for this purpose they follow the threshold strategy explained in the previous section. To obtain the transmission cost threshold $e^{*}$ for this system, we first calculated the empirical CDF $F_{K}(x)$, which is the CDF of the minimum of $\mathrm{K}$ identically distributed random variables whose $\mathrm{CDF}$ is $F(x)$. Using $F_{K}(x)$ we numerically obtained the transmission probability at the Nash equilibrium, $p^{*}$, as shown in figure 1 . Then we used $e^{*}=\left(1-p^{*}\right)^{N-1}$ to obtain the transmission cost threshold for the selfish system.

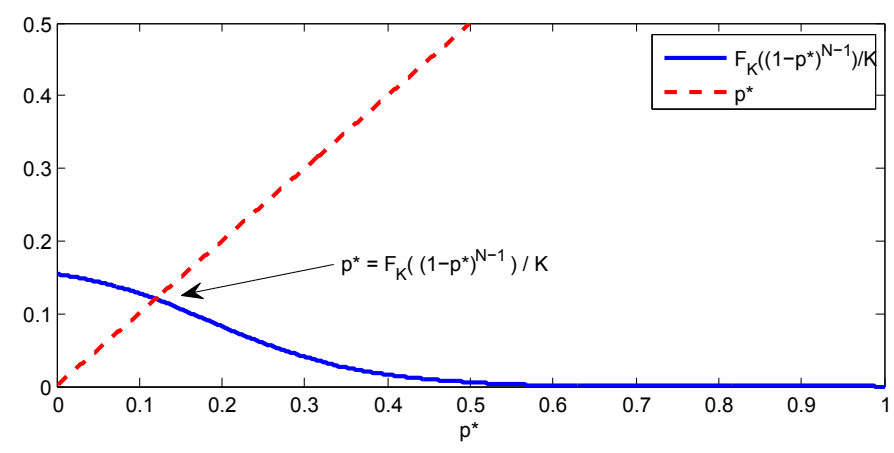

Fig. 1. Obtaining the transmission probability at Nash equilibrium numerically according to (8).

Similar to the selfish system, the users in the cooperative system transmit their packets when their transmission costs are below a threshold, but their objective is to maximize the expected sum utility in the system. As seen in figure 2 , as transmission cost threshold increases from 0 to 1 the probability of successful transmission initially increases, then decreases due to increasing number of collisions. On the other hand, the expected transmission cost increases monotonously with increasing threshold. Thus, the maximum utility is obtained when the difference between success probability and expected transmission cost is maximum. When simulating the cooperative system, we use the transmission cost threshold that maximizes this difference.

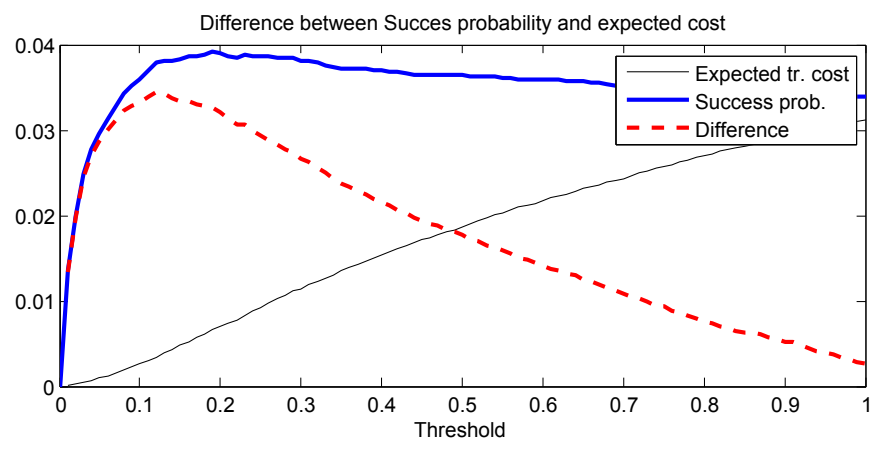

Fig. 2. Probability of success and expected transmission cost versus transmission threshold in the cooperative system.

As in the case of the cooperative system, users in the scheduling system try to maximize the sum utility. In our simulations of the scheduling system, we obtained the transmission schedule which maximizes the sum utility by means of an exhaustive search.

In the cooperative system with one cheater, the cooperating users follow the same transmission strategy as the purely cooperative system (i.e. they use the same threshold). On 
the other hand, the selfish user is aware of the cooperative users' strategy, therefore it adjusts its threshold accordingly: Using the cooperative system's threshold $e_{\text {coop }}^{*}$, the cheater first obtains the transmission probability of cooperative users, which is given by $p_{\text {coop }}^{*}=F_{K}\left(e_{\text {coop }}^{*}\right)$. Using this probability, it calculates the threshold that maximizes its utility in the cooperative system. Namely, the cheater uses $e_{\mathrm{ch}}^{*}=\left(1-p_{\text {coop }}^{*}\right)^{N-1}$ as its transmission cost threshold.

\section{Results}

Figure 3 shows the average sum utility per channel in the three systems. As expected, the performance of the scheduling system is the best of the three because in this system the users, who have complete information on channel conditions, choose to transmit or wait such that in every slot the highest sum utility is achieved. As the number of users in the system increases, probability of finding a user with a smaller transmission cost also increases due to greater diversity. Therefore, average sum utility per channel approaches 1 as the number of users increases in the scheduling system. However, if we compare this to the cooperative system, we observe that the performance decreases as much as $60 \%$. This drop in performance is due to users' lack of complete information; in the cooperative system the users transmit probabilistically, therefore collisions occur to the detriment of system performance. If the users are acting selfishly instead, then they decrease their transmission cost thresholds in order to increase their transmission probability at the prospect of obtaining a better utility. This, however, leads to even greater number of collisions and the system performance degrades further. This degradation becomes more pronounced as the load in the system $(N / K)$ increases. In a loaded system the price of anarchy is as much as $400 \%$.

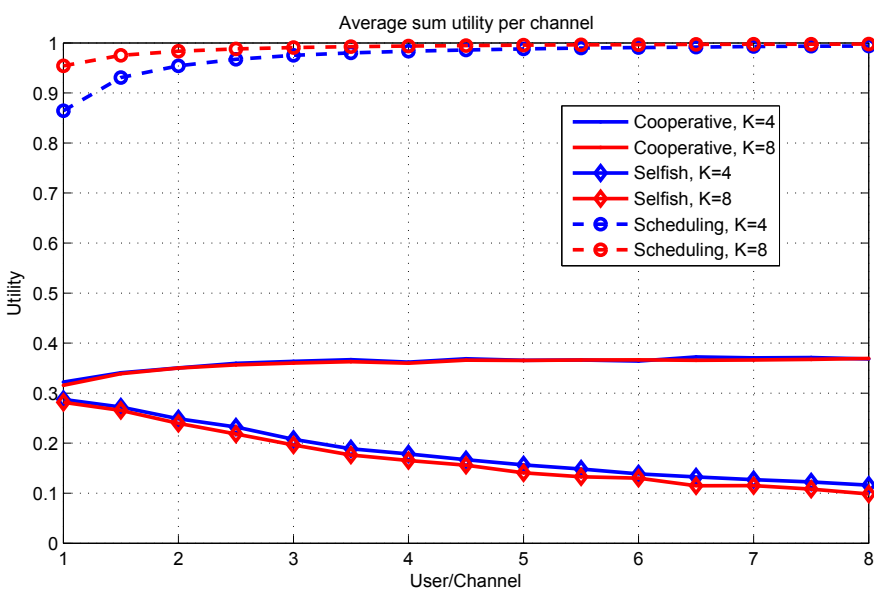

Fig. 3. Average sum utility per channel for two systems with 4 and 8 channels and up to 32 and 64 users.

From the user's point of view, average user utility decreases as the load increases, as shown in figure 4 , which generally applies to all four systems. With respect to this metric, the scheduling system is again the best performer, approaching an average user utility of $1 / N$, followed by the cooperative system and the selfish system. The performance of these three systems relative to each do not show variation as load increases. However, if we consider that one user in the cooperative system starts to act selfishly, then this cheating user obtains a significant gain in utility. As system load increases, the cheater's relative gain in the cooperative system also increases, eventually surpassing even the average utility of a user in the scheduling system.

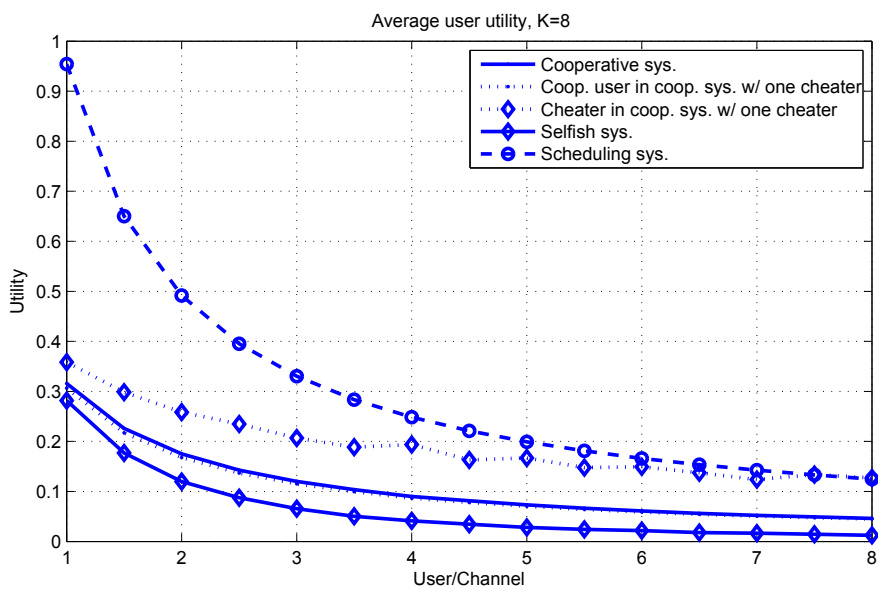

Fig. 4. Average user utility in the scheduling, cooperative and selfish systems where there are 8 channels and up to 64 users.

The increase in the selfish user's utility can be seen more clearly in figure 5. In this figure, all the represented systems have 8 channels and 64 users, and the utilities of two users within these systems are plotted against each other. The point D indicates the utility of two users in the scheduling system. Note that both users receive the same average utility because their objectives are the same. If the users lack complete information but are still acting cooperatively, then their utilities will end up in B. If, however, one of the users (user 2 in the figure) were to start acting selfishly, this cheating user would obtain much higher utility, while the other user (user 1 in the figure) like all cooperative users, would suffer a small decrease in its average utility. This change corresponds to $\mathrm{C}$ and the mentioned decrease can also be observed in figure 4, where the utility of cooperating users in a cooperative system with one selfish user is slightly smaller than the utility of users in a purely cooperative system.

The observation that cheating gives a prospect of obtaining much higher utility in a cooperative system creates great incentive for users of the cooperative system to start acting selfishly. On the other hand, if all of the users in the system would start acting selfishly, then the utility of all users would decrease drastically, and the user utilities would end up in A. The decrease in utility from $\mathrm{B}$ to $\mathrm{A}$ is the price paid for anarchy in the system, which approaches $400 \%$ as load increases in the system.

\section{CONCLUSIONS}

In this paper we analyzed the performance of selfish and cooperative multichannel random access systems under the condition that the users have incomplete information about 


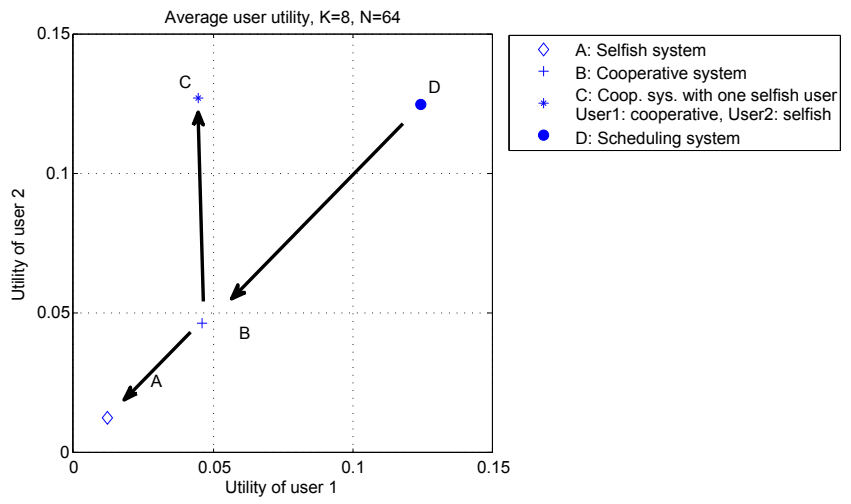

Fig. 5. Comparison of utilities of two users in the scheduling, cooperative and selfish systems where there are 8 channels and 64 users in the system.

the system. We showed that under this condition the selfish users' behavior will be in the form of a threshold strategy, that is, they will transmit if their minimum transmission cost is less than a certain threshold and wait otherwise, and we derived this threshold analytically. Our results showed that lack of complete information causes a degradation of up to $60 \%$ in average utility in a cooperative system. Furthermore, we observed that there is a large incentive for individual users to behave selfishly in a cooperative MRA system. At the same time, the loss in average utility is not significant when only one user acts selfishly. However, when a significant number of (or all) users start acting selfishly, the drop in average user utility, "the price of anarchy", is significant. This loss in performance in our examples approaches $75 \%$, as user load increases.

For future work we plan to incorporate the effects of capture in our analysis, and design algorithms to drive the operating point of the selfish system to a desired (social) optimum.

\section{REFERENCES}

[1] I. F. Akyildiz, W.-Y. Lee, M. C. Vuran, and S. Mohanty, "Next generation/dynamic spectrum access/cognitive radio wireless networks: A survey," Computer Networks, vol. 50, no. 13, pp. 2127 - 2159, 2006.

[2] C. Ngo and V. Li, "Fixed channel assignment in cellular radio networks using a modified genetic algorithm," Vehicular Technology, IEEE Transactions on, vol. 47, no. 1, pp. 163-172, Feb 1998.

[3] H. Inaltekin and S. Wicker, "The analysis of nash equilibria of the oneshot random-access game for wireless networks and the behavior of selfish nodes," Networking, IEEE/ACM Transactions on, vol. 16, no. 5, pp. 10941107, Oct. 2008.

[4] A. MacKenzie and S. Wicker, "Selfish users in aloha: A game-theoretic approach," in IEEE VTS 54th Vehicular Technology Conference, 2001. VTC 2001 Fall, vol. 3, 2001.

[5] J. Hultell, "Cooperative and non-cooperative wireless access," Ph.D. dissertation, Royal Institute of Technology (KTH), Dec. 2008.

[6] D. Wang, C. Comaniciu, H. Minn, and N. Al-Dhahir, "A game-theoretic approach for exploiting multiuser diversity in cooperative slotted aloha," Wireless Communications, IEEE Transactions on, vol. 7, no. 11, pp. 4215-4225, November 2008.

[7] W. Szpankowski, "Packet switching in multiple radio channels: Analysis and stability of a random access system," Computer Networks (1976), vol. 7, no. 1, pp. $17-26,1983$.

[8] D. Bertsekas and R. Gallager, Data networks (2nd ed.). Upper Saddle River, NJ, USA: Prentice-Hall, Inc., 1992. 